\title{
Heavy Quark Phenomenology from Lattice QCD
}

\author{
Damir Becirevic ${ }^{\mathrm{a} *}$ \\ ${ }^{\text {a} D i p . ~ d i ~ F i s i c a, ~ U n i v e r s i t a ̀ ~ " L a ~ S a p i e n z a " ~ a n d ~ I N F N, ~ P i a z z a l e ~ A l d o ~ M o r o ~ 2, ~ I-00185 ~ R o m e, ~ I t a l y ~}$
}

Recent results relevant for the $B$-physics phenomenology, obtained from lattice QCD simulations by the APE Collaboration, are reviewed. This includes the $B^{0}-\bar{B}^{0}$ mixing amplitude, $B \rightarrow \pi$ semileptonic decay and the relative width difference of $B_{s}^{0}$ mesons, $(\Delta \Gamma / \Gamma)_{B_{s}}$.

The main theoretical obstacles in determining the amount of $\mathrm{CP}$-violation that comes from the Standard Model (SM) are related to the uncertainties in computation of various hadronic quantities. In this talk, I focus on several such quantities/processes involving heavy-light mesons, for which APE group provided new lattice results. Technical details about the simulations are given in the references which will be quoted with each quantity I discuss in this paper. Here I only stress that all our results are obtained at $\beta=6.2$, in the quenched approximation, and by using the (fully propagating) $\mathcal{O}(a)$ improved, Wilson fermions.

\section{Decay Constants $\left(f_{B}\right.$ and others) [ 1, 2]}

One of the essential hadronic quantities entering the $B_{q}^{0}-\bar{B}_{q}^{0}$ mixing amplitude is the $B$-meson decay constant $f_{B_{q}}(q=d, s)$, defined as

$\left\langle 0\left|\bar{b} \gamma_{\mu} \gamma_{5} q\right| B_{q}(p)\right\rangle=i p_{\mu} f_{B_{q}}$.

The central results from our two simulations (see Tab. (1) are obtained by: (i) linearly extrapolating (interpolating) $f_{H_{q}} \sqrt{m_{H_{q}}}$ in $1 / m_{H_{q}}$, to the $B$ $(D)$ meson mass; (ii) including the KLM factor in a way discussed in [ [3]; (iii) converting to the physical units by using $a^{-1}\left(m_{K^{*}}\right)=2.7(1) \mathrm{GeV}$. To estimate the systematic errors, we combine in quadrature the following differences between our central values and the ones obtained when: (a) extrapolating in $1 / m_{H_{q}}$ quadratically; (b) ommiting the KLM factor, (c) using the ratio $f_{H} / f_{\pi}$ to extrapolate to $m_{B}$. Since the extrapolation from the directly accessed heavy meson masses $\left(2 \mathrm{GeV} \leq m_{H} \leq 3 \mathrm{GeV}\right)$ to $m_{B}$ is rather long,

\footnotetext{
*Talk given at "Lattice 2000", Bangalore, India. Based on works done with A. Abada, Ph.Boucaud, V.Giménez, L.Giusti, J.P.Leroy, V.Lubicz, G.Martinelli, D.Meloni, F.Mescia and A. Retico.
}

the source (a) largely dominates the systematics. This error has not been included in the results [2], where only 3 heavy quarks were considered. Note, however, that this error completely cancels in the $\mathrm{SU}(3)$ breaking ratio, $f_{B_{s}} / f_{B_{d}}$. For comparison with results of other lattice groups, see [ [4]. In [

Table 1

Results for pseudoscalar decay constants in MeV. First errors are statistical and the second are systematic.

\begin{tabular}{c|c|c}
\hline \hline & Ref. [1]] & Ref. [2] \\
\hline$f_{B}$ & $173(13)_{-3}^{+26}$ & $175(22)_{-0}^{+8}$ \\
$f_{B_{s}} / f_{B}$ & $1.14(2)(1)$ & $1.17(4)(1)$ \\
$f_{D}$ & $216(11)(5)$ & $207(11)_{-0}^{+4}$ \\
$f_{D_{s}} / f_{D}$ & $1.11(1)(1)$ & $1.13(3)(1)$ \\
\hline \hline
\end{tabular}

3. we have also computed the vector meson decay constants, which I now update. In addition we compute the coupling of the vector meson to the tensor current, i.e.

$\left\langle 0\left|\bar{b} \gamma_{\mu} q\right| B_{q}^{*}(p, \lambda)\right\rangle=i e_{\mu}^{(\lambda)} m_{B_{q}^{*}} f_{B_{q}^{*}}$

$\left\langle 0\left|\bar{b} \sigma_{\mu \nu} q\right| B_{q}^{*}(p, \lambda)\right\rangle=i\left(e_{\mu}^{(\lambda)} p_{\nu}-p_{\mu} e_{\nu}^{(\lambda)}\right) f_{B_{q}^{*}}^{T}(\mu)$,

where $\mu$ is the scale at which the tensor density is non-perturbatively renormalized (in the RI-MOM scheme). These decay constants are particularly important in testing the validity of the factorization in non-leptonic decays of heavy-light mesons. Our new results are

$$
\begin{aligned}
& f_{B^{*}}=199(14)_{-4}^{+34} \mathrm{MeV} ; f_{D^{*}}=258(14)(6) \mathrm{MeV} ; \\
& f_{B_{s}^{*}} / f_{B^{*}}=1.14(2)(1) ; f_{B^{*}}^{T}\left(m_{b}\right) / f_{B^{*}}=0.88(2)(2) ; \\
& f_{D_{s}^{*}} / f_{D^{*}}=1.10(2) ; f_{D^{*}}^{T}(2 \mathrm{GeV}) / f_{D^{*}}=0.90(2)
\end{aligned}
$$




\section{2. $\mathbf{B}_{\mathbf{q}}^{0}-\overline{\mathbf{B}}_{\mathbf{q}}^{0}$ Mixing and $(\Delta \Gamma / \Gamma)_{\mathbf{B}_{\mathbf{s}}}[2,10$}

To access any bare continuum $\Delta B=2$ operator from the lattice, by using Wilson fermions, one first has to subtract the effect of mixing with other dimension-six 4-fermion operators which is due to the explicitely broken chiral symmetry in the Wilson quark action. A bare (continuum) operator should then be appropriately renormalized. The whole procedure can be shortly written as

$$
\begin{aligned}
& \left\langle\bar{B}_{q}^{0}\left|Q_{i}(\mu)\right| B_{q}^{0}\right\rangle= \\
& \left\langle\bar{B}_{q}^{0}\left|\sum_{j} Z_{i j}\left(g_{0}^{2}, \mu\right)\left(Q_{j}^{\text {latt. }}+\sum_{k \neq j} \Delta_{k}\left(g_{0}^{2}\right) Q_{k}^{\text {latt. }}\right)\right| B_{q}^{0}\right\rangle,
\end{aligned}
$$

where $i, j, k$ run over the basis of parity conserving operators $\left(Q_{i}\right), \Delta_{i}\left(g_{0}^{2}\right)$ and $Z_{i j}\left(g_{0}^{2}, \mu\right)$ are the subtraction and renormalization (RC) constants, respectively. A technique to compute the constants $\Delta_{i}$ and $Z_{i j}$ non-perturbatively, in the RIMOM renormalization scheme, has been developed in ref. [ 5]. We work in Landau gauge, apply the technique [5] at three different values of the scale $\mu$, and verify that the scale dependence of $Z_{i j}(\mu)$, for the operators discussed below, is indeed well described by the perturbative NLO anomalous dimension matrix [6]. This allows us to express our matrix elements in the renormalization group invariant (RGI) form. 2

$B_{q}^{0}-\bar{B}_{q}^{0}$ Mixing: The needed parameter, $B_{B_{q}}$ $(q=d, s)$, is defined as

$\left\langle\bar{B}_{q}^{0}\left|Q_{L}(\mu)\right| B_{q}^{0}\right\rangle=\frac{8}{3} m_{B_{q}}^{2} f_{B_{q}}^{2} B_{B_{q}}(\mu)$,

where $Q_{L}=\bar{b}^{i} \gamma_{\mu}\left(1-\gamma_{5}\right) q^{i} \bar{b}^{j} \gamma_{\mu}\left(1-\gamma_{5}\right) q^{j}$, and $i, j$ are the color indices. From the definitions (11) and (21), it is clear that the $B$-parameter can be directly accessed if (for each heavy light-meson $H_{q}$ ) we compute the ratio of correlation functions

$$
\begin{aligned}
\frac{(3 / 8) \cdot \sum_{\vec{x}, \vec{y}}\left\langle P(x) Q_{L}(0 ; \mu) P^{\dagger}(y)\right\rangle}{\sum_{\vec{x}}\left\langle P(x) A_{0}^{\dagger}(0)\right\rangle \sum_{\vec{y}}\left\langle A_{0}(0) P^{\dagger}(y)\right\rangle} \stackrel{-t_{x}, t_{y} \gg 0}{\longrightarrow} & B_{H_{q}}(\mu) .
\end{aligned}
$$

The last limit is valid when the operator $Q_{L}$ and the pseudoscalar sources $P$ are sufficiently separated on the temporal axis. To reach the physically relevant $B_{B_{q}}$ from the extracted $B_{H_{q}}$ (which

2 The procedure sketched above can be highly simplified if one uses the Ward identity to relate the parity conserving to the parity violating operators (for which $\Delta_{i}=0$ ) [ This idea is yet to be implemented in practice. should scale with heavy meson mass as a constant), we make the linear $1 / m_{H}$ fit and extrapolate to $m_{B}$. Our final results are

$$
\begin{gathered}
\hat{B}_{B_{d}}=1.38(11)_{-.09}^{+.00}, \quad \hat{B}_{B_{s}}=1.35(5)_{-.08}^{+.00}, \\
\hat{B}_{B_{s}} / \hat{B}_{B_{d}}=0.98(5),
\end{gathered}
$$

where we converted (to NLO) the directly computed $B_{B_{q}}^{\mathrm{RI}-\mathrm{MOM}}(\mu)$ to the RGI form $\hat{B}_{B_{q}}$. We also obtained, $\hat{B}_{D}=1.24(4)_{-.09}^{+.00}$, which may be useful in the non-SM phenomenology. Since we clearly see $1 / m_{H}^{(n)}$ corrections from our data, one can try to constrain the extrapolation by using the static result for $\hat{B}_{B}$ [8]. Such an exercise leads to a $\sim 5 \%$ lower value of $B_{B}$, which is well within our error

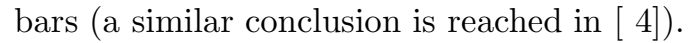

After combining the above results with the ones for $f_{B_{q}}$, also computed in [2], we get

$$
\begin{aligned}
& f_{B_{d}} \sqrt{\hat{B}_{B_{d}}}=206(28)(7) \mathrm{MeV} ; \\
& \xi \equiv \frac{f_{B_{s}}}{f_{B_{d}}} \sqrt{\frac{\hat{B}_{B_{s}}}{\hat{B}_{B_{d}}}}=1.16(7) .
\end{aligned}
$$

In the last result, most of the systematic uncertainties cancel in the ratio. To exemplify the phenomenological benefit of this result, I combine our value for $\xi$ with the updated experimental value for the frequency of $B_{d}^{0}$ mesons oscilations $\left(\Delta m_{d}^{(\text {exp. })}=0.486(15) \mathrm{ps}^{-1}[\right.$ 9]), to get

$$
\begin{aligned}
\Delta m_{s} & =\frac{\left|V_{t s}\right|^{2}}{\left|V_{t d}\right|^{2}} \xi^{2}\left(\frac{m_{B_{s}}}{m_{B_{d}}} \Delta m_{d}\right)^{\text {(exp.) }} \\
& =16.2 \pm 2.1 \pm 3.4 \mathrm{ps}^{-1}
\end{aligned}
$$

where $\left|V_{t s}\right|^{2} /\left|V_{t d}\right|^{2}=24.4 \pm 5.0$ is assumed. Experimental lower bound is $\Delta m_{s}^{\text {(exp.) }}>14.9 \mathrm{ps}^{-1}$ [ []] $(\Delta \Gamma / \Gamma)_{B_{s}}$ : In the framework of the heavy quark expansion, the leading contribution in the expression for the width difference of $B_{s}^{0}$ mesons, comes from $\Delta B=2$ operator, $Q_{S}=\bar{b}^{i}\left(1-\gamma_{5}\right) s^{i} \bar{b}^{j}(1-$ $\left.\gamma_{5}\right) s^{j}$, the matrix element of which is parameterized as

$\left\langle\bar{B}_{s}^{0}\left|Q_{S}(\mu)\right| B_{s}^{0}\right\rangle=-\frac{5}{3}\left(\frac{m_{B_{s}}^{2} f_{B_{s}}}{m_{b}(\mu)+m_{s}(\mu)}\right)^{2} B_{S}(\mu)(7)$

where $B_{S}(\mu)$ is the wanted bag parameter. An important observation made in ref. [10] is that if we only replace $O_{L} / 8 \rightarrow-O_{S} / 5$ in eq. (3), we see a very large dependence on the inverse heavy meson 
mass $1 / m_{H_{s}}$. On the contrary, if in denominator of eq. (3) we also replace the axial current by the pseudoscalar density, $A_{0} \rightarrow P$, the $1 / m_{H_{s}}$ dependence becomes much weaker and the extrapolation to $m_{B}$ is more under control (which is why our central results are those obtained using the latter procedure). Obviously, the large $1 / m_{H}$ dependence comes from the ratio of the heavy meson/heavy quark mass, $\left[m_{H_{s}} /\left(m_{Q}+m_{s}\right)\right]^{2}$. It will be interesting to see whether the inconsistency of the two procedures disappears with the simulations performed closer to $m_{B_{s}}$. In this calculation, we also needed to compute the matrix element of the operator $\tilde{Q}_{S}$ which is the one obtained by reversing the color inidices in $Q_{S}$, and which mix with $Q_{S}$ in the continuum. Once we extract the values for $B_{S}(\mu)$ and $\tilde{B}_{S}(\mu)$, we converted RI-MOM $\rightarrow \overline{\mathrm{MS}}$ since the Wilson coefficients, $G(z)$ and $G_{S}(z)\left(z=m_{c}^{2} / m_{b}^{2}\right)$, were computed in the $\overline{\mathrm{MS}}$ scheme [11]. After a linear extrapolation to $1 / m_{B_{s}}$, we get

$B_{S}^{\overline{\mathrm{MS}}}\left(m_{b}\right)=0.86(2)(3) ; \tilde{B}_{S}^{\overline{\mathrm{MS}}}\left(m_{b}\right)=1.25(3)_{-.05}^{+.02}$.

For the physical prediction of $(\Delta \Gamma / \Gamma)_{B_{s}}$, one needs the ratio of the matrix elements (2) and (7). We obtain

$\mathcal{R}^{\overline{\mathrm{MS}}}\left(\mu=m_{b}\right)=-0.93(3)(1)$

which is in a good agreement with results obtained by using the effective theories [ 8,12$]$. We proposed in [10] a safer way to predict

$$
\begin{aligned}
\left(\frac{\Delta \Gamma}{\Gamma}\right)_{B_{s}} & =K\left(\tau_{B_{s}} \Delta m_{B_{d}} \frac{m_{B_{s}}}{m_{B_{d}}}\right)^{\text {(exp. })} \times \\
& \left(G(z)-G_{S}(z) \mathcal{R}\left(m_{b}\right)+\tilde{\delta}_{1 / m}\right) \xi^{2}
\end{aligned}
$$

where $K$ is the known constant and $\tilde{\delta}_{1 / m}$ encodes $1 / m_{b}$ corrections (which are estimated by using the vacuum saturation approximation). The advantage of using this formula is that it contains experimentally well determined quantities, and $\xi$ and $\mathcal{R}\left(m_{b}\right)$ ratios, in which (again) most of the systematic uncertainties cancel. Finally, we obtained

$$
\begin{aligned}
\left(\frac{\Delta \Gamma}{\Gamma}\right)_{B_{s}}= & {\left[(0.5 \pm 0.1)-(13.8 \pm 2.8) \mathcal{R}\left(m_{b}\right)\right.} \\
& \left.+(15.7 \pm 2.8) \tilde{\delta}_{1 / m}\right] \cdot 10^{-2} \\
= & (4.7 \pm 1.5 \pm 1.6) \cdot 10^{-2}
\end{aligned}
$$

where we show how the explicit cancellation occurs between the leading $\mathcal{R}\left(m_{b}\right)$ and the $1 / m_{b}$ correcting terms. Therefore, to improve the above result it is necessary to gain a better control over the dimension-seven operators which appear in $\tilde{\delta}_{1 / m}$.

\section{3. $\left|V_{u b}\right|$ from $B(B \rightarrow \pi \ell \nu)$ [13]}

Compared to "Lattice 99" [ [1], we now have the final results for the $D$ decay modes (see [13]), and also for the most challenging one, $B \rightarrow \pi$. The relevant form factors, $F_{+/ 0}\left(q^{2}\right)\left(q=p_{H}-p\right)$,

$$
\begin{array}{r}
\left\langle\pi(p)\left|\bar{q} \gamma_{\mu} Q\right| H\left(p_{H}\right)\right\rangle=\frac{m_{H}^{2}-m_{\pi}^{2}}{q^{2}} q_{\mu} F_{0}\left(q^{2}\right)+ \\
\left(p_{H}+p-q \frac{m_{H}^{2}-m_{\pi}^{2}}{q^{2}}\right)_{\mu} F_{+}\left(q^{2}\right),
\end{array}
$$

are extracted for 3 different light $(q)$ and 4 heavy $(Q)$ quark masses and for 13 inequivalent kinematical configurations $\left(\vec{p}_{H}, \vec{q}\right)$. The mass extrapolations of form factors are known to be trickier because of the interplay between $m_{H}$ and $q^{2}$ dependences. A parameterization for the $q^{2}$-dependence, which contains most of the theoretically available constraints, has been proposed in [14]:

$$
\begin{aligned}
& F_{+}\left(q^{2}\right)=\frac{c_{H}\left(1-\alpha_{H}\right)}{\left(1-\tilde{q}^{2}\right)\left(1-\alpha_{H} \tilde{q}^{2}\right)}, \\
& F_{0}\left(q^{2}\right)=\frac{c_{H}\left(1-\alpha_{H}\right)}{\left(1-\tilde{q}^{2} / \beta_{H}\right)},
\end{aligned}
$$

where $\tilde{q}^{2}=q^{2} / m_{B^{*}}^{2}$. The parameters $\phi \in$ $\left\{c_{H} \sqrt{m_{H}},\left(1-\alpha_{H}\right) m_{H},(\beta-1) m_{H}\right\}$ should scale as a constant (plus corrections $\propto 1 / m_{H}$ ). To reach $B \rightarrow \pi$ we first fit the form factors to the parameterization (12) for each combination of the heavy and light quarks and then adopted the following two methods:

Method I We smoothly extrapolate to the final pion (kaon) state, for every heavy quark, and then use the scaling laws for all three parameters to extrapolate to $B$, namely

$\phi=a_{0}+\frac{a_{1}}{m_{H}}+\frac{a_{2}}{m_{H}^{2}}$.

Method II Following the proposal of UKQCD group [15], we first extrapolate to the final pion at fixed

$v \cdot p=\frac{M_{H_{d}}^{2}+M_{\pi}^{2}-q^{2}}{2 M_{H_{d}}}$, 
Table 2

APE lattice results for the $B \rightarrow \pi$ form factors [13] are compared to the predictions obtained by using LCSR [116].

\begin{tabular}{cccc}
\hline \hline & Method I & Method II & LCSR [16] \\
\hline$c_{B}$ & $0.42(13)(4)$ & $0.51(8)(1)$ & $0.41(12)$ \\
$\alpha_{B}$ & $0.40(15)(9)$ & $0.45(17)_{-.13}^{+.06}$ & $0.32_{-.07}^{+.21}$ \\
$\beta_{B}$ & $1.22(14)_{-.03}^{+.12}$ & $1.20(13)_{-.00}^{+.15}$ & - \\
\hline$F(0)$ & $0.26(5)(4)$ & $0.28(6)(5)$ & $0.28(5)$ \\
$F^{B \rightarrow K}(0) / F^{B \rightarrow \pi}(0)$ & $1.21(9)_{-.09}^{+.00}$ & $1.19(11)_{-.11}^{+.03}$ & $1.28(11)_{-.10}^{+.18}$ \\
$F_{0}\left(m_{B}^{2}\right)$ & $1.3(6)_{-.4}^{+.0}$ & $1.5(5)_{-.4}^{+.0}$ & - \\
$g_{B^{*} B \pi}$ & $20 \pm 7$ & $24 \pm 6$ & $22 \pm 7$ \\
\hline$\left|V_{u b}\right|^{-2} \Gamma\left(B^{0} \rightarrow \pi^{-} \ell^{+} \nu\right)\left[\mathrm{ps}^{-1}\right]$ & $6.3 \pm 2.4 \pm 1.6$ & $8.5 \pm 3.8 \pm 2.2$ & $7.3 \pm 2.5$ \\
\hline \hline
\end{tabular}

and then extrapolate to $B$ by using the HQET scaling laws. The $q^{2}$-behavior is then fit by using eq. (12).

The results obtained by using both methods are shown in Tab. 2, where we also make a comparison with the light cone QCD sum rules (LCSR) predictions [16]. Besides a very good consistency of the results of the two (different) methods, a pleasant feature of this analysis is also the agreement with lattice results of [15] as well as with the LCSR results [16]. For a further comparison with presently available lattice results, see [ [4].

The central values in Tab. 2 are obtained through a quadratic extrapolation to $m_{B}$, which provides a better consistency with the CallanTreiman relation, $\left.F_{0}\left(m_{B}^{2}\right)=f_{B} / f_{\pi} \cdot\right]^{3}$ The complete account of the systematic uncertainties is detailed in [ 13]. I emphasize that $F^{B \rightarrow \pi}(0)$ in Tab. 2, as obtained after extrapolating $F^{H \rightarrow \pi}(0) m_{H}^{3 / 2}$ to $m_{B}$, is indistinguishible from the one obtained by combining separately extrapolated $c_{B}$ and $\alpha_{B}$ into $F^{B \rightarrow \pi}(0)=c_{B}\left(1-\alpha_{B}\right)$.

To read the content of the parameterization (12) I recall that the parameter $c_{B}$ measures the residue of $F_{+}\left(q^{2}\right)$ at $m_{B^{*}}^{2}$, thus allowing determination of the $g_{B^{*} B \pi}$. By using the standard definitions, one has $g_{B^{*} B \pi}=2 m_{B^{*}} c_{B} / f_{B^{*}}$ and the results are also given in Tab. 2. The parameter $\alpha_{B}$ indicates that besides the first pole $\left(q^{2}=m_{B^{*}}^{2}\right)$, all

${ }^{3}$ Research on verification of this relation on the lattice has been discussed at this conference [17]. the other singularities contributing to $F_{+}\left(q^{2}\right)$ can be mimicked by an effective pole corresponding to $m_{1-} \simeq 8 \pm 1 \mathrm{GeV}$, whereas the contributions to the scalar form factor $F_{0}\left(q^{2}\right)$ are represented by an effective pole with the mass $m_{0^{+}} \simeq 6 \pm 3 \mathrm{GeV}$.

Finally, by comparing our results for the decay width, with the experimental branching ratio $B\left(\bar{B}^{0} \rightarrow \pi^{+} \ell \bar{\nu}\right)$, we obtain $\left|V_{u b}\right|=(4.1 \pm 1.1) 10^{-3}$.

\section{REFERENCES}

1. A. Abada et al., hhep-lat/9910021].

2. D. Becirevic et al., [hep-lat/0002025] ].

3. D. Becirevic et al., [hep-1at/9811003].

4. C. Bernard, hep-lat/0011064].

5. A. Donini et al., [hep-lat/9902030].

6. M. Ciuchini et al., [hep-ph/9711402];

A. J. Buras et al., [hep-ph/0005183].

7. D. Becirevic et al., [hep-lat/0005013].

8. V. Giménez, J. Reyes, [hep-lat/0010048].

9. A. Stocchi, hep-ph/0010222 .

10. D. Becirevic et al., [hep-ph/0006135].

11. M. Beneke et al., [hep-ph/9808385].

12. S. Hashimoto, et al., [hep-lat/0004022].

13. A. Abada et al., hhep-lat/0011065].

14. D. Becirevic, A.Kaidalov, [hep-ph/990449d] .

15. K. C. Bowler et al., hhep-lat/9911011]

16. A. Khodjamirian et al., [hep-ph/0001297] .

17. S. Aoki et al., hep-lat/0011008;

C. Maynard, hhep-lat/0010016]. 